\title{
EVALUACIÓN DE LA ADAPTABILIDAD Y ESTABILIDAD DE 14 HÍBRIDOS DE MAÍZ, AZUERO, PANAMÁ1
}

\author{
Román Gordón-Mendoza ${ }^{2}$, Ismael Camargo-Buitrago ${ }^{3}$, Jorge Franco-Barrera ${ }^{4}$, Andrés González-Saavedra 4
}

\begin{abstract}
RESUMEN
Evaluación de la adaptabilidad y estabilidad de 14 híbridos de maíz, Azuero, Panamá. Se sembró un ensayo a través de nueve ambientes contrastantes de la Región de Azuero durante el ciclo agrícola 2005-2006, con el objetivo de seleccionar los híbridos de maíz con mayor estabilidad y adaptabilidad en esta región. El material genético consistió de 14 híbridos blancos y amarillos. Se utilizó el diseño experimental de bloques completos al azar con tres repeticiones, las parcelas experimentales consistieron de dos surcos de 5,2 m de largo, separadas a 0,8 m. Se realizó un análisis de varianza combinado y las medias se separaron utilizando la DMS. Para estimar la adaptabilidad y estabilidad de híbridos y ambientes, se usó el modelo AMMI y la técnica GGE Biplot-SREG. Los análisis estadísticos indicaron diferencias significativas $(\mathrm{P}<0,01)$ entre genotipos, ambientes y la interacción GenotipoAmbiente, indicando la respuesta diferencial de los híbridos ante los diferentes ambientes. El híbrido PB-0105 sobresalió del resto de los híbridos con un promedio de 8,01 t/ha. A este genotipo le siguieron el grupo de híbridos formados por 30S-40, PB-0103, P-0512, P-0102, 30F-80 y DK-466 con rendimientos superiores a las 7,0 t/ha. El análisis de estabilidad identificó al híbrido P-0102 como el más estable. El análisis de la precipitación durante el desarrollo del cultivo, definió tres grupos de localidades en relación al estrés sufrido en la fase de floración (51-80 dds) y llenado del grano ( $>80 \mathrm{dds})$. El grupo de tres localidades que no fue afectado por el estrés hídrico superó en 1,21 y 2,02t/ha a los otros dos grupos ambientales.
\end{abstract}

Palabras clave: Maíz, híbridos, Biplot GGE-SREG, estabilidad, efecto de precipitación pluvial.

\begin{abstract}
Adaptability and stability assessment of 14 corn hybrids, Azuero, Panama. An experiment was established across nine contrasting environments in Azuero during 2005-2006, to select corn hybrids with the best stability and adaptability on the Azuero Region. The genetic materials consisted in 14 white and yellow corn hybrids. A Completely Randomized Block Design with three replications was used. The experimental plots consisted of two rows of $5.2 \mathrm{~m}$ of length, separated by $0.8 \mathrm{~m}$. A combined Analysis of Variances was done and the means was separated by LSD. To estimate adaptability and stability of hybrids and environments, AMMI and GGE Bi-plot and SREG technique were used. The statistical analysis indicated significant differences $(\mathrm{P}<0.01)$ among Genotypes, Environments and the Genotype-Environment Interaction, indicating the differential response of hybrids confronted with different environments. The hybrid P-0105 was superior to all others, with mean yield of $8.01 \mathrm{t} / \mathrm{ha}$. This genotype was followed by the hybrid group formatted by 30S-40, PB-0103, P-0512, P-0102, 30F-80 and DK-466 with yields above $7 \mathrm{t} / \mathrm{ha}$. The stability analysis identified the hybrid P-0102 like the most stable. The analysis of rainfall measured during crop development this year, defined three locality groups related with the flowering phase (51-80 dap) and grain fill phase (>80 dap) stress suffered. The group of three localities without water stress surpassed in 1.21 and $2.02 \mathrm{t} / \mathrm{ha}$ the other two environments groups.
\end{abstract}

Key words: Corn, hybrids, Biplot GGE-SREG, stability, rainfall effect.

1 Recibido: 2 de mayo, 2006. Aceptado: 17 de julio, 2006.

2 Instituto de Investigación Agropecuaria de Panamá (IDIAP), CIA Azuero, "Ing. Germán De León”, Los Santos, Panamá, Email: gordon.roman@gmail.com

3 Instituto de Investigación Agropecuaria de Panamá (IDIAP), CIA Recursos Genéticos "Ing. Alfonso Alvarado", Río Hato, Panamá Email: icamargo@cwpanama.net

4 Instituto de Investigación Agropecuaria de Panamá (IDIAP), CIA de Azuero “Ing. Germán De León”, Los Santos, Panamá. 


\section{INTRODUCCIÓN}

La selección de genotipos que interaccionen lo menos posible con el ambiente, ha sido uno de los principales objetivos en los programas de mejoramiento genético tanto de las instituciones estatales como de las empresas que se dedican a la venta de germoplasma. La evaluación de genotipos a través de distintos ambientes, principalmente en ambientes contrastantes, es una de las prácticas más usuales para la recomendación de nuevos materiales a los productores de una región o zona específica. La Interacción Genotipo-Ambiente (IGA) ocurre cuando hay respuestas diferentes de los genotipos en relación con la variación del ambiente. Esta interacción merece gran importancia en la evaluación de híbridos desarrollados para diferentes circunstancias de producción, es necesario integrar los conceptos de adaptabilidad y estabilidad para definir el comportamiento de genotipos evaluados a través de ambientes contrastantes. La adaptabilidad se refiere a la capacidad de los genotipos de aprovechar ventajosamente los estímulos del ambiente, en cuanto que la estabilidad se refiere a la capacidad de los genotipos de mostrar un comportamiento altamente previsible en función del estímulo ambiental. Por otro lado, independientemente de la metodología empleada para estimar la IGA, hay que tener claro a cual concepto de estabilidad se refiere. Becker (1981); Lin et al. (1986); Becker y León (1988), definen conceptos de estabilidad fenotípica que se complementan del punto de vista estadístico, biológico y agronómico.

Allard y Hansche (1969); citados por Márquez (1991), definen a una variedad estable como una variedad con capacidad de amortiguamiento o flexibilidad para cambiar en actitud, que para el caso de variedades agrícolas, significaría ajustar su rendimiento a las condiciones ambientales, es decir, variedades capaces de ajustar sus procesos vitales para mantener la productividad. Por otro lado Simmonds (1962), citado por Marquez (1991), resalta que el término adaptabilidad se ha tomado como la capacidad para responder a la selección, lo cual implica la variabilidad genética. En estas condiciones una población genotípicamente heterogénea será adaptable a diferentes ambientes al estar sujeta a diferentes presiones de selección, manifestando su adaptación específica a un ambiente de acuerdo a la presión de selección de éste, mediante su respuesta cambiante a los diferentes ambientes, se medirá la adaptabilidad.
El análisis de varianza y regresión conjunta, es una metodología empleada ampliamente para explicar la IGA (Finlay y Wilkinson 1963; Eberhart y Russell 1966; Perkins y Jinks 1968). Técnicas multivariadas también han sido usadas para estudiar los efectos de la IGA; por ejemplo, el análisis de componentes principales (PCA), análisis de coordenadas principales, análisis de cluster (Crossa 1990; Westcott 1986). El desarrollo del modelo AMMI (Efectos principales aditivos e interacción multiplicativa), que integra análisis de varianza y de componentes principales (Zobel et al. 1988), ha mostrado su eficiencia para explicar una proporción de la suma de cuadrados de la interacción, superior a la obtenida con el análisis de varianza y regresión conjunta (Gauch y Zobel 1988; Zobel et al. 1988; Crossa 1988; Crossa 1990; Crossa et al. 1990; Crossa et al. 1991). El presente trabajo se realizó con el objetivo de identificar híbridos promisorios, con buena estabilidad de rendimiento y características agronómicas deseables, bajo diversos ambientes de Panamá; además se buscó determinar la adaptabilidad y estabilidad de 14 híbridos comerciales y experimentales de grano amarillo y blanco generados por IDIAP y compañías privadas en nueve ambientes de la Región de Azuero.

\section{MATERIALES Y MÉTODOS}

\section{Ubicación}

El experimento se estableció en nueve localidades de la Región de Azuero, en parcelas facilitadas por productores de maíz de las provincias de Herrera y Los Santos, durante la segunda época de siembra del año 2005 (Cuadro 1).

\section{Características edáficas}

Antes de la siembra de los ensayos se obtuvieron varias muestras de suelo para su análisis físico-químico en cada localidad. Las mismas se tomaron en cada uno de los bloques a una profundidad de $0-20 \mathrm{~cm}$. Estas fueron homogenizadas y enviadas al Laboratorio de Suelos del IDIAP, en donde se les realizó el análisis según Díaz-Romeu y Hunter (1978). El análisis preliminar de suelo de los sitios, mostró la variabilidad natural que se encuentra en la región maicera de Azuero, en donde los suelos varían de muy ácidos $(\mathrm{pH} 4,4)$ a poco 
Cuadro 1. Ubicación, fecha de siembra y cosecha del ensayo de evaluación de híbridos de maíz establecido en nueve localidades. Azuero, Panamá, 2005.

\begin{tabular}{lllcllc}
\hline No. & Localidad & Distrito & Latitud & Longitud & Fecha de siembra & Fecha de cosecha \\
\hline & Herrera & & & & & \\
1 & Las Cabras & Pesé & $7^{\circ} 52.597^{\prime}$ & $80^{\circ} 32.745^{\prime}$ & $14-09-05$ & $13-01-06$ \\
& Los Santos & & & & & \\
2 & El Ejido & Los Santos & $7^{\circ} 54.488^{\prime}$ & $80^{\circ} 22.307^{\prime}$ & $31-08-05$ & $13-01-06$ \\
3 & Sabana Grande & Los Santos & $7^{\circ} 50.197^{\prime}$ & $80^{\circ} 22.382^{\prime}$ & $20-09-05$ & $12-01-06$ \\
4 & Guararé & Guararé & $7^{\circ} 49.149^{\prime}$ & $80^{\circ} 17.436^{\prime}$ & $09-09-05$ & $06-01-06$ \\
5 & La Enea & Guararé & $7^{\circ} 51.082^{\prime}$ & $80^{\circ} 16.467^{\prime}$ & $02-09-05$ & $04-01-06$ \\
6 & Las Cocobolas & Las Tablas & $7^{\circ} 47.024^{\prime}$ & $80^{\circ} 14.976^{\prime}$ & $05-09-05$ & $05-01-06$ \\
7 & Paraíso & Pocrí & $7^{\circ} 41.001^{\prime}$ & $80^{\circ} 08.350^{\prime}$ & $08-09-05$ & $05-01-06$ \\
8 & Pocrí & Pocrí & $7^{\circ} 39.145^{\prime}$ & $80^{\circ} 07.186^{\prime}$ & $13-09-05$ & $11-01-06$ \\
9 & Mariabé & Pedasí & $7^{\circ} 33.154^{\prime}$ & $80^{\circ} 02.965^{\prime}$ & $15-09-03$ & $10-01-06$ \\
\hline
\end{tabular}

ácidos (pH 6,5), con texturas que van de franco-arenosos hasta franco-arcillosos. En cuanto a la fertilidad, todos son bajos en fósforo con excepción de las localidades en Sabana Grande y Guararé, medio en Potasio, bajos en Aluminio y altos en Calcio y Magnesio. El porcentaje de materia orgánica es considerado bajo y el mismo osciló entre 1,07 a 2,22\% (Cuadro 2).

\section{Características climáticas}

Se tomaron datos de precipitación pluvial en ocho pluviómetros ubicados en zonas adyacentes a los ensayos. La precipitación pluvial registrada durante el año
2005 fue de $1.616,0 \mathrm{~mm}$ y la misma está por encima del promedio del período 1995-2005. En los meses previos a la siembra de los ensayos de este año (abrilagosto), se registró una precipitación acumulada promedio de $812,3 \mathrm{~mm}$, mientras que en el período de septiembre a diciembre la media registrada fue de $803,7 \mathrm{~mm}$; ambos valores están por encima de la media de la precipitación del período 1995-2005 (626,8 y 633,6 $\mathrm{mm}$, respectivamente).

Durante el mes de septiembre, se registró una precipitación por debajo del promedio anual de la Región (211 mm) en las localidades de París, El Ejido, Ciénega Larga y Guararé, mientras que en las otras cuatro se

Cuadro 2. Principales características físico químicas de los suelos donde se efectuaron los ensayos, Azuero, Panamá, 2005.

\begin{tabular}{|c|c|c|c|c|c|c|c|c|c|c|c|c|c|c|c|}
\hline \multirow[t]{2}{*}{ Localidad } & \multirow{2}{*}{ Arena } & \multirow{2}{*}{$\begin{array}{r}\text { Limo } \\
(\%)\end{array}$} & \multirow[t]{2}{*}{ Arcilla } & \multirow[t]{2}{*}{ pH } & $\mathbf{P}$ & $\mathbf{K}$ & $\mathbf{C a}$ & Mg & Al & \multirow{2}{*}{$\frac{\text { M.O. }}{(\%)}$} & Mn & $\mathbf{F e}$ & $\mathbf{Z n}$ & $\mathrm{Cu}$ & \multirow[t]{2}{*}{ Text } \\
\hline & & & & & \multicolumn{2}{|c|}{$(\mu \mathrm{g} / \mathrm{ml})$} & \multicolumn{3}{|c|}{$(\mathrm{meq} / \mathbf{1 0 0 m l})$} & & \multicolumn{4}{|c|}{$(\mu \mathrm{g} / \mathrm{ml})$} & \\
\hline El Ejido & 54 & 18 & 28 & 4,9 & 2 & 86 & 6,5 & 4,2 & 0,3 & 2,22 & 54 & 14 & 1 & 2 & FARA \\
\hline La Enea & 68 & 20 & 12 & 4,4 & 8 & 82 & 1,4 & 2,0 & 0,7 & 1,28 & 56 & 22 & 9 & 1 & FA \\
\hline Cocobolas & 38 & 22 & 40 & 5,1 & 8 & 102 & 6,4 & 6,0 & 0,3 & 1,07 & 55 & 15 & 1 & 7 & FARC \\
\hline Paraíso & 34 & 26 & 40 & 4,7 & 2 & 188 & 11,2 & 11,1 & 0,2 & 1,57 & 38 & 15 & 1 & 2 & FARC \\
\hline Guararé & 48 & 20 & 32 & 6,1 & 42 & 156 & 23,0 & 4,6 & 0,1 & 1,47 & 36 & 27 & 1 & 1 & FARA \\
\hline Pocrí & 40 & 32 & 28 & 5,8 & 7 & 167 & 8,8 & 4,0 & 0,7 & 1,76 & 32 & 27 & 1 & 2 & FARC \\
\hline Las Cabras & 40 & 32 & 28 & 5,0 & 5 & 82 & 13,1 & 5,6 & 0,5 & 1,57 & 109 & 20 & 1 & 1 & FARC \\
\hline Mariabé & 36 & 32 & 32 & 5,4 & 1 & 141 & 9,0 & 4,9 & 0,1 & 1,17 & 48 & 22 & 1 & 4 & FARC \\
\hline \multicolumn{16}{|l|}{ Sabana } \\
\hline Grande & 56 & 26 & 18 & 6,5 & 226 & 239 & 18,5 & 5,8 & 0,2 & 1,37 & 26 & 20 & 2 & 1 & FA \\
\hline
\end{tabular}

M.O. = Materia orgánica; Text $=$ Textura $;$ FARC $=$ franco arcilloso, FARA $=$ franco arcillo-arenoso, FA $=$ Franco arenoso 
observaron precipitaciones por encima de éste. El mes de octubre registró lluvias dentro los parámetros normales en la mayoría de las localidades, con excepción de Pocrí, que presentó un registro de 502,1 mm. El mes de noviembre tuvo similar comportamiento que el mes de septiembre con registros por debajo de la media $(251,4 \mathrm{~mm})$ en las localidades ubicadas al norte de la Península. Cabe destacar, que la media de este mes fue superior al promedio de los últimos once años en donde se lleva el registro de estos mismos pluviómetros. Diciembre se caracterizó por presentar un incremento en la precipitación, principalmente de los pluviómetros ubicados en Pocrí y Pedasí, en las otras localidades la precipitación fue menor que la media de todas las localidades, excepto Ciénega Larga que registró valores que no difieren del promedio general del año (Cuadro 3). De manera general los cuatro pluviómetros ubicados en los distritos ubicados al norte de la península (Parita, Los Santos y Guararé) presentaron registros por debajo del promedio ( $803,7 \mathrm{~mm}$ ) en el período de desarrollo del cultivo (septiembre-diciembre), mientras que los ubicado más al sur (Las Tablas, Pocrí y Pedasí) los registros superaron los $800 \mathrm{~mm}$ en el mismo período.

\section{Material genético}

Los híbridos evaluados fueron obtenidos de diferentes fuentes. Las compañías privadas suministraron ocho genotipos, mientras que el sector oficial contribuyó con seis. El ensayo incluyó once genotipos con grano de color amarillo y tres de color blanco (Cuadro 4).
Cuadro 4. Nombre, tipo, color del grano y origen de híbridos de maíz evaluados. Azuero, Panamá, 2005.

\begin{tabular}{rllll}
\hline No. & Híbrido & $\begin{array}{c}\text { Color del } \\
\text { grano }\end{array}$ & Tipo & Origen \\
\hline & & Amarillo & Simple & Pioneer \\
1 & 30 K-75 & Amarillo & Simple & Pioneer \\
2 & $30 \mathrm{~F}-80$ & Amarillo & Simple & Pioneer \\
3 & 30 P-70 & Amarillo & Triple & Dekalb \\
4 & DK-466 & Amarillo & Triple & Pioneer \\
5 & 30 S-40 & Amarillo & Triple & Pioneer \\
6 & $30 F-87$ & Amarillo & Doble & Pioneer \\
7 & 3031 & Blanco & Doble & Pioneer \\
8 & $30 R-92$ & Blanco & Triple & IDIAP \\
9 & PB-0103 & Blanco & Triple & IDIAP \\
10 & PB-0105 & Amarillo & Triple & IDIAP \\
11 & P-0408 & Amarillo & Triple & IDIAP \\
12 & P-0512 & Amarillo & Triple & IDIAP \\
13 & P-0102 & Amarillo & Triple & IDIAP \\
14 & P-0104 & Amp &
\end{tabular}

\section{Manejo agronómico}

El sistema de preparación del suelo de los experimentos fue el convencional; el mismo consistió en dar de dos a tres pases de rastra pesada hasta dejar el suelo desmenuzado. La densidad inicial de siembra utilizada fue de 6,25 plantas $/ \mathrm{m}^{2}$, ésto se logró al sembrar en surcos separados a $0,80 \mathrm{~m}$ y dejar una planta cada $0,20 \mathrm{~m}$. La fertilización consistió en la aplicación de $227 \mathrm{~kg} / \mathrm{ha}$ de la fórmula química 12-24-12 al momento de la

Cuadro 3. Precipitación pluvial (mm) en ocho pluviómetros de la Región de Azuero, Panamá 2005.

\begin{tabular}{|c|c|c|c|c|c|c|c|c|c|c|}
\hline Localidad & París & El Ejido & $\begin{array}{l}\text { Ciénega } \\
\text { Larga }\end{array}$ & Guararé & $\begin{array}{l}\text { Tablas } \\
\text { Abajo }\end{array}$ & $\begin{array}{c}\text { Las } \\
\text { Cocobolas }\end{array}$ & Pocrí & Pedasí & $\begin{array}{c}\text { Prom. } \\
2005\end{array}$ & $\begin{array}{r}\text { Prom. } \\
95-05\end{array}$ \\
\hline Abr & 35,0 & 16,0 & 35,0 & 9,7 & 1,4 & 5,0 & 0,0 & 7,4 & 27,2 & 13,8 \\
\hline May & 116,0 & 171,0 & 106,5 & 144,5 & 141,7 & 286,9 & 240,0 & 171,5 & 162,6 & 119,3 \\
\hline Jun & 273,0 & 247,0 & 182,0 & 127,0 & 244,9 & 197,8 & 232,1 & 248,0 & 230,7 & 149,0 \\
\hline Jul & 55,0 & 102,0 & 131,5 & 142,3 & 205,6 & 275,0 & 378,5 & 345,9 & 194,6 & 175,9 \\
\hline Ago & 107,0 & 113,0 & 105,5 & 133,5 & 163,8 & 199,6 & 247,4 & 604,7 & 197,1 & 164,0 \\
\hline Sep & 201,0 & 131,0 & 115,5 & 170,2 & 228,4 & 217,0 & 318,0 & 305,4 & 211,0 & 171,3 \\
\hline Oct & 260,0 & 205,0 & 218,0 & 210,4 & 317,9 & 222,8 & 502,1 & 217,8 & 259,4 & 214,0 \\
\hline Nov & 143,0 & 193,0 & 212,0 & 124,6 & 325,6 & 311,6 & 362,1 & 395,1 & 251,4 & 182,7 \\
\hline Dic & 14,0 & 30,0 & 80,0 & 25,8 & 61,9 & 76,9 & 266,5 & 144,6 & 81,8 & 65,6 \\
\hline Total & $1.204,0$ & $1.208,0$ & $1.186,0$ & $1.088,0$ & $1.691,2$ & $1.792,6$ & $2.546,7$ & $2.440,4$ & $1.616,0$ & $1.260,3$ \\
\hline Abr-Ago & 586,0 & 649,0 & 560,5 & 557,0 & 757,4 & 964,3 & 1098,0 & 1377,5 & 812,3 & 626,8 \\
\hline Sep-Dic & 618,0 & 559,0 & 625,5 & 531,0 & 933,8 & 828,3 & 1448,7 & 1062,9 & 803,7 & 633,6 \\
\hline
\end{tabular}


siembra, en forma de banda continua a $4 \mathrm{~cm}$ de la línea de siembra. Posteriormente, se realizaron dos aplicaciones suplementarias de urea, la primera a los 20 días después de siembra (dds) a razón de $159 \mathrm{~kg} / \mathrm{ha}$ y la segunda a los 37 dds a razón de $204 \mathrm{~kg} / \mathrm{ha}$. En total se aplicó $194 \mathrm{~kg} \mathrm{~N}, 54 \mathrm{~kg}$ de $\mathrm{P}_{2} \mathrm{O}_{5}$ y $27 \mathrm{~kg} \mathrm{~K} 2 \mathrm{O} / \mathrm{ha}$.

El control de malezas consistió en la aplicación en pre-emergencia de la mezcla de atrazina más pendimentalina a razón de 1,5 y $1,65 \mathrm{~kg}$ i.a./ha, respectivamente. En algunas localidades, por la presencia de malezas de mayor tamaño, se añadió a la mezcla $1,84 \mathrm{~kg}$ i.a./ha de glifosato. En la localidad de Las Cabras se realizó una aplicación suplementaria a los 12 dds del herbicida halosulfurón metil en dosis de 60 g i.a./ha para el control del Cyperus rotundus.

\section{Variables medidas}

Los datos tomados incluyeron caracteres cuantitativos, que son altamente influenciados por el ambiente como: altura de planta y mazorca, rendimiento de grano, porcentaje de plantas acamadas, porcentaje de mazorcas podridas y caracteres cualitativos, que son pocos influenciados por el ambiente, como: días a floración femenina, número de plantas y mazorcas al momento de la cosecha, humedad del grano y la evaluación de las principales enfermedades al follaje (Curvularia sp. y Puccinia sp.). Para la evaluación de enfermedades se utilizó una escala de 1 a 5 , donde 1 indica ausencia de enfermedad y 5 infección muy severa. La evaluación se realizó en las etapas finales (70-80 dds) del cultivo, antes que las hojas se tornaran de color café.

\section{Diseño experimental}

Las parcelas experimentales consistieron de dos surcos de 5,2 m de largo. Para la ejecución en campo se utilizó el diseño de bloques completos al azar, con tres repeticiones, de acuerdo al siguiente modelo matemático:

$$
X_{I J L}=\mu+G_{I}+B_{J / L}+A_{L}+(G A)_{I L}+e_{I J K}
$$

En donde:

$\mathrm{X}_{\mathrm{IJL}}=$ Valor del carácter estudiado

$\mu=$ Media general

$\mathrm{G}_{\mathrm{I}}=$ Efecto de Genotipo

ISSN: 1021-7444
$\mathrm{B}_{\mathrm{J} / \mathrm{L}}=$ Efecto de bloques dentro de repetición

$\mathrm{A}_{\mathrm{L}}=$ Efecto de ambiente

$(\mathrm{G} \mathrm{A})_{\mathrm{IL}}=$ Efecto de la interacción Genotipo Ambiente

$\mathrm{e}_{\mathrm{IJK}}=$ Error Experimental

\section{Análisis estadístico}

Se realizó un análisis de varianza combinado, considerando un modelo mixto (ambiente aleatorio y genotipo fijo). Para la separación de medias de rendimiento se aplicó la diferencia mínima significativa (DMS). Para el análisis de las variables porcentaje de plantas acamadas y porcentaje de mazorcas podridas, los valores fueron transformados por el método de la raíz cuadrada más un medio $(\sqrt{ } \mathrm{x}+0,5)$.

\section{Análisis de estabilidad}

Para el análisis de estabilidad se utilizó el modelo AMMI Biplot GGE-SREG, que integra el análisis de varianza y el análisis de componentes principales (Zobel et al. 1988; Yan et al. 2000). El modelo matemático es:

$$
\mathrm{Y}_{\mathrm{ge}}=\mu+\alpha_{\mathrm{g}}+\beta_{\mathrm{e}}+\Sigma^{\mathrm{N}} \lambda_{\mathrm{n}} \mathrm{Y}_{\mathrm{gn}} \delta_{\mathrm{en}}+\rho_{\mathrm{ge}}
$$

En donde:

$\mathrm{Y}_{\mathrm{g} \mathrm{e}}=$ Rendimiento promedio de un genotipo $\mathrm{g}$ en un ambiente e

$\mu=$ Media general

$\lambda_{\mathrm{n}}=$ Es el valor singular para el PCA

$\mathrm{N}=$ Número de PCA retenidos en el modelo

$\mathrm{Y}_{\mathrm{gn}}=$ Son los valores de vectores de los genotipos (PCA)

$\alpha_{\mathrm{g}}=$ Efecto de las desviaciones de las medias de los genotipos

$\beta_{\mathrm{e}}=$ Efecto de las desviaciones de las medias del ambiente

$\delta_{\mathrm{en}}=$ Son los valores de los vectores para cada ambiente (PCA)

$\rho_{\mathrm{g} \mathrm{e}}=$ Residual

\section{RESULTADOS Y DISCUSIÓN}

\section{Análisis de varianza}

En el Cuadro 5 se presenta el resumen de los análisis de varianza combinados del rendimiento y

AGRONOMÍA MESOAMERICANA 17(2): 189-199. 2006 
Cuadro 5. Cuadrados medios y significancia encontrada en el análisis de varianza combinado del rendimiento y otras características agronómicas evaluadas en el ensayo regional de maíz. Azuero, Panamá. 2005.

\begin{tabular}{|c|c|c|c|c|c|c|c|c|c|c|c|c|}
\hline F. de V & g.l. & Rend & AlPt & $\mathbf{P t m}^{2}$ & $\mathrm{Mzm}^{2}$ & $\mathrm{Mz} / \mathrm{pt}$ & PMz & Aca & Pud & Roya & Curv & $\begin{array}{c}\text { AlMz/ } \\
\text { AlPt }\end{array}$ \\
\hline Ambiente & 8 & $41,57 * *$ & $16174 * *$ & $1,20 * *$ & $1,00 *$ & $0,013^{* *}$ & $14845^{* *}$ & $0,237 * *$ & $0,0011 * *$ & $1,875^{* *}$ & $3,59 * *$ & $0,026^{* *}$ \\
\hline Rep (Amb) & 18 & 2,55 & 833,7 & 0,13 & 0,23 & 0,002 & 500,1 & 0,006 & 0,0003 & 0,206 & 0,19 & 0,002 \\
\hline Genotipo & 13 & $3,93 * *$ & $3252,5^{* *}$ & $0,46 * *$ & $0,56 * *$ & $0,008 * *$ & $1045,9 * *$ & $0,086 * *$ & $0,0014 * *$ & $1,773 * *$ & $0,76^{* *}$ & $0,012^{* *}$ \\
\hline Gen $x$ Amb & 104 & $1,34 * *$ & $138,2 * *$ & $0,16 * *$ & $0,27 * *$ & $0,002^{\text {n.s. }}$ & $289,5^{* *}$ & $0,012 * *$ & $0,0003 * *$ & $0,161 * *$ & $0,11 * *$ & $0,001 * *$ \\
\hline Error & 234 & 0,55 & 101,5 & 0,06 & 0,12 & 0,002 & 138,7 & 0,003 & 0,0002 & 0,079 & 0,068 & 0,001 \\
\hline C.V. $(\%)$ & & 10,5 & 4,2 & 4,3 & 5,9 & 4,6 & 9,6 & 7,2 & 2,1 & 10,7 & 10,5 & 5,8 \\
\hline
\end{tabular}

$* *, *$ diferencias estadísticas con probabilidad menor al 1, $5 \%$, n.s.= Efecto no significativo.

Rend $=$ Rendimiento de grano, AlPt $=$ Altura de planta, $\mathrm{Ptm}^{2}=$ Plantas por metro cuadrado, Mzm² $=\mathrm{Mazorcas}^{2}$ por metro cuadrado,

$\mathrm{Mz} / \mathrm{Pt}=$ Mazorcas por planta, PMz = Peso de mazorcas, Aca = Porcentaje de plantas acamadas, Pud = Porcentaje mazorcas podridas, Cur = Curvularia, AlMz/AlPt = Relación altura de mazorca y altura de planta.

algunas características agronómicas, donde se observa que hubo diferencia altamente significativa entre ambientes, híbridos y la interacción de ambos factores en todas las variables evaluadas, excepto en la interacción genotipo ambiente de la variable mazorcas por planta.

\section{Efecto de ambientes}

De acuerdo al análisis de varianza el ambiente capturó el 47,7\% de la suma de cuadrados total del experimento. El Cuadro 6 presenta el rendimiento de grano y algunas de las variables medidas por

Cuadro 6. Fecha de siembra, promedio de rendimiento y otras características agronómicas de la Prueba Regional de maíz según localidades, Azuero, Panamá, 2005.1,2

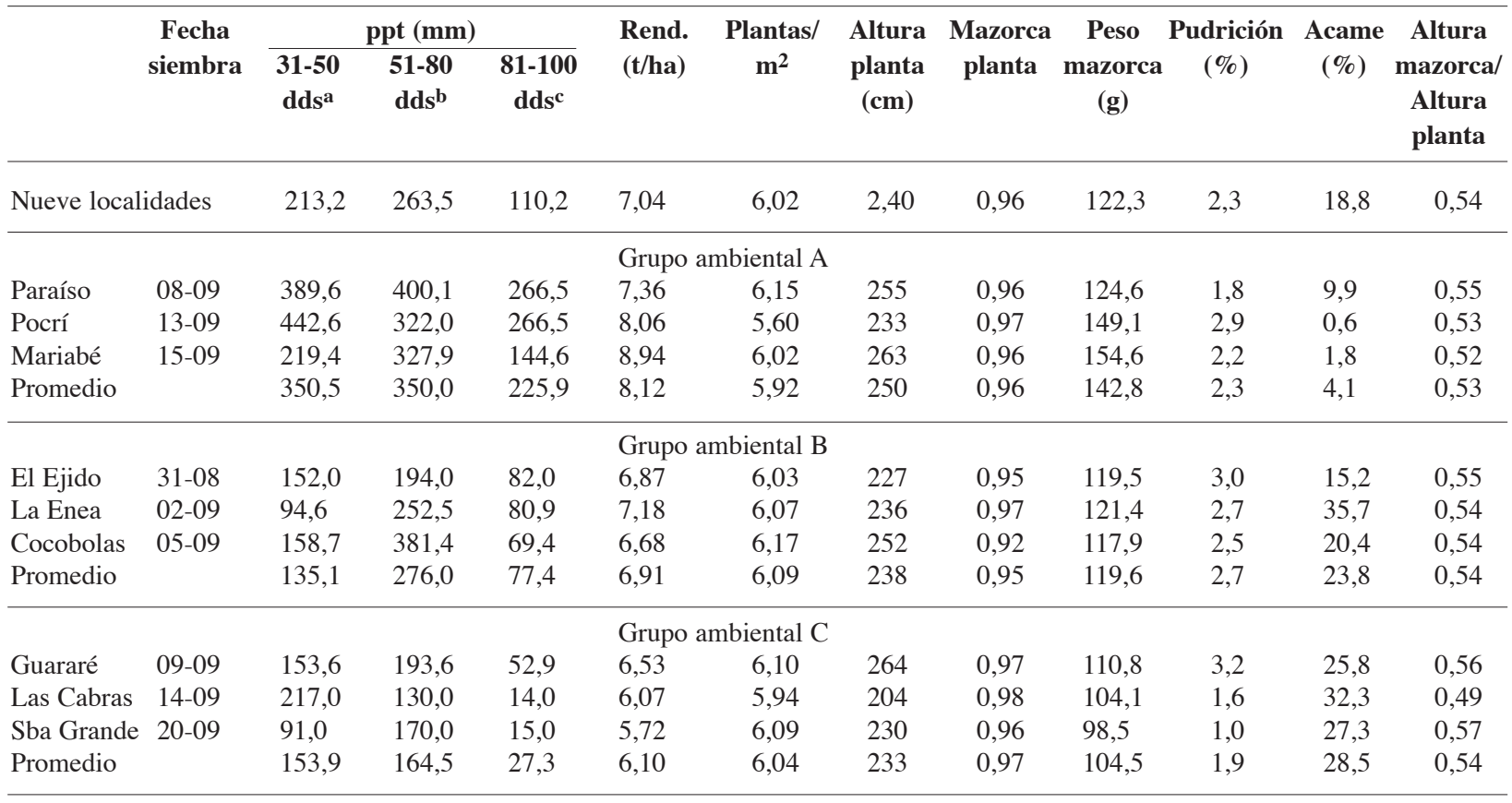

\footnotetext{
${ }^{1} \mathrm{ppt}=$ precipitación, ${ }^{2} \mathrm{dds}=$ días después de siembra; ${ }^{\mathrm{a}}$ Pre floración; ${ }^{\mathrm{b}}$ Floración/llenado; ${ }^{\mathrm{c}}$ Llenado de grano.
} 
localidad. El rendimiento promedio a través de las nueve localidades fue de 7,04 t/ha, éste fue afectado por la distribución de las lluvias en las distintas etapas fenológicas del cultivo. Algunas localidades fueron afectadas por estrés hídrico en la etapa de floración (51-80 dds) y en mayor medida durante la fase de llenado del grano (81-100 dds) a pesar de que en general el promedio del período en que se desarrolló el cultivo fue superior al promedio del período 1995-2005.

La información generada en el ensayo permitió identificar tres grupos ambientales o dominios de recomendación. El primer grupo (Grupo ambiental A) lo conformaron las localidades ubicadas al sur de la península como lo son Paraíso, Pocrí y Mariabé; este grupo tuvo en común una precipitación promedio de 350,0 y $225,9 \mathrm{~mm}$ en las fases de floración/llenado (51-80 dds) y llenado del grano (>80 dds), respectivamente. El segundo grupo (Grupo ambiental B) agrupó los ensayos sembrados en El Ejido, La Enea y Las Cocobolas con precipitaciones acumuladas de 276,0 y $77,4 \mathrm{~mm}$ en las fases analizadas. Estas localidades están ubicadas en una zona con mayor probabilidad de sufrir estrés hídrico en las últimas fases del cultivo, cuando se siembra después del 15 de septiembre (Gordón et al. 2004); estos registros representan una reducción de 21,2 y $65.7 \%$ en las fases analizadas al compararse con las localidades del Grupo A. El tercer grupo (Grupo ambiental C) estuvo formado por los ensayos sembrados en Guararé, Sábana Grande y Las Cabras, los mismos están ubicados geográficamente en la misma zona que los ensayos del Grupo B; al ser sembrados los ensayos más tarde, el registro de lluvias fue de 164,5 y $27,3 \mathrm{~mm}$ en dos de las tres fases críticas del cultivo. Estos promedios representan una reducción de 53,0 y $87,9 \%$ en relación al registro del Grupo A y de 40,4 y 64,7\% menos lluvia con respecto al Grupo B. La baja precipitación pluvial en la fase floración/llenado en las localidades del Grupo B, se tradujo en rendimientos menores de 7,2 t/ha y pesos de mazorcas menores de $122 \mathrm{~g}$, lo que representó en promedio una reducción del rendimiento de grano de $14.9 \%(1,21 \mathrm{t} / \mathrm{ha})$ con respecto a los ensayos del grupo A. En los ensayos del Grupo $\mathrm{C}$ el estrés hídrico experimentado en las dos fases representó una mayor merma en el rendimiento, la cual fue de $11,7 \%(0,80$ t/ha) con respecto al Grupo B y de $24.8 \%$ (2,02 t/ha) en relación al promedio del Grupo A.

En relación a las variables plantas por metro cuadrado, relación entre las alturas de mazorca, número de mazorcas por planta y el porcentaje de mazorcas podridas no fueron afectadas por las diferencias presentadas entre los tres grupos ambientales. La variable porcentaje de plantas acamadas varió grandemente entre los tres grupos, observándose un incremento en el porcentaje a medida que la localidad fue sometida a un mayor estrés, esto se puede explicar a que mayor estrés, la planta presenta un menor vigor lo que se traduce en una mayor propensión al acame. El otro factor que se vio afectado fue la altura de planta, de manera que a mayor estrés en las localidades, menor tamaño de planta provocado por el acortamiento de los entrenudos.

\section{Efecto de genotipos}

El análisis de varianza mostró diferencias altamente significativas entre los distintos híbridos evaluados para la variable rendimiento de grano; logrando capturar el 7,3\% de la suma de cuadrados del análisis de varianza de esta variable. El Cuadro 7 muestra la media de rendimiento de las nueve localidades, así como el comportamiento de los híbridos en los tres grupos ambientales definidos.

De los híbridos evaluados, siete superaron en rendimiento a la media general, sobresaliendo de manera significativa el híbrido PB-0105 con media de 8,01 t/ha. Siguieron a este genotipo el grupo formado por los híbridos 30S-40, PB-0103, P-0512, P-0102, 30F-80 y DK-466 con rendimientos promedio superiores a 7 $\mathrm{t} / \mathrm{ha}(7,38 ; 7,30 ; 7,24 ; 7,19 ; 7,16$ y $7,10 \mathrm{t} / \mathrm{ha}$, respectivamente). Un tercer grupo de híbridos estuvo conformado por 30K-75, P-0104, P-0408, y 30P-70, los cuales presentaron rendimientos promedios arriba de las 6,77 t/ha. Se observó que los híbridos más afectados por el déficit hídrico fueron 30S-40, P-0102, DK-466 y 30F-87, con mermas superiores a las 2,50 t/ha. Mientras que los menos afectados fueron los híbridos con el menor promedio 3031 y 30R-92 con reducciones menores de 0,56 y $1,07 \mathrm{t} / \mathrm{ha}$.

En cuanto a la población de plantas, al momento de la cosecha no se encontró diferencias estadísticas significativas entre ninguno de los híbridos mencionados, lo que sugiere que las diferencias en rendimientos son debidas al potencial de rendimiento y adaptabilidad de cada uno de ellos y no de la cantidad de plantas cosechadas. En relación a las otras características evaluadas, los promedios obtenidos para cada una de las variables a través de las nueve localidades se 
Cuadro 7. Rendimiento, población y peso de mazorca de los híbridos evaluados, Azuero, Panamá, 2005.

\begin{tabular}{|c|c|c|c|c|c|c|c|c|c|c|c|c|}
\hline \multirow[t]{2}{*}{ Híbridos } & \multicolumn{4}{|c|}{ Rendimiento (t/ha) } & \multicolumn{4}{|c|}{ Plantas $/ \mathbf{m}^{2}(\%)$} & \multicolumn{4}{|c|}{ Peso mazorcas (g) } \\
\hline & 9 loc. & Gr-A & Gr-B & Gr-C & 9 loc. & Gr-A & Gr-B & Gr-C & 9 loc. & Gr-A & Gr-B & Gr-C \\
\hline PB-0105 & 8,01 & $\mathbf{9 , 5 3}$ & 7,35 & 7,14 & 6,13 & 6,06 & 6,18 & 6,13 & 136,2 & 160,8 & 126,9 & 121,0 \\
\hline $30 S-40$ & 7,38 & 9,00 & $\mathbf{7 , 7 6}$ & 5,39 & 6,08 & 5,94 & 6,26 & 6,04 & 124,7 & 156,0 & 125,5 & 92,6 \\
\hline PB-0103 & 7,30 & 8,65 & 6,62 & 6,63 & 5,98 & 5,88 & 6,04 & 6,02 & 128,0 & 154,1 & 115,7 & 114,2 \\
\hline P-0512 & 7,24 & 8,14 & 6,77 & 6,81 & 6,13 & 6,09 & 6,18 & 6,13 & 125,1 & 140,6 & 118,9 & 115,6 \\
\hline P-0102 & 7,19 & 8,69 & 6,96 & 5,91 & 5,88 & 5,90 & 5,81 & 5,93 & 126,1 & 150,8 & 126,1 & 101,4 \\
\hline 30F-80 & 7,16 & 7,64 & 7,59 & 6,25 & 6,07 & 5,90 & 6,17 & 6,13 & 122,9 & 135,7 & 128,7 & 104,2 \\
\hline DK-466 & 7,10 & 8,36 & 7,33 & 5,60 & 5,97 & 5,85 & 6,08 & 6,00 & 124,6 & 147,8 & 127,7 & 98,2 \\
\hline $30 \mathrm{~K}-75$ & 6,91 & 7,86 & 6,82 & 6,06 & 6,14 & 6,01 & 6,22 & 6,18 & 117,5 & 135,4 & 116,9 & 100,3 \\
\hline P-0104 & 6,88 & 7,90 & 7,31 & 5,45 & 5,84 & 5,75 & 6,13 & 5,65 & 120,2 & 138,9 & 121,3 & 100,5 \\
\hline P-0408 & 6,79 & 7,62 & 6,32 & 6,42 & 6,03 & 6,06 & 5,96 & 6,06 & 122,3 & 136,2 & 119,0 & 111,8 \\
\hline $30 \mathrm{P}-70$ & 6,77 & 8,13 & 6,33 & 5,85 & 6,12 & 6,23 & 6,02 & 6,12 & 112,4 & 133,4 & 105,4 & 98,4 \\
\hline $30 F-87$ & 6,73 & 7,90 & 6,99 & 5,30 & 6,06 & 6,10 & 6,14 & 5,94 & 114,5 & 134,0 & 117,8 & 91,7 \\
\hline 30R-92 & 6,59 & 6,99 & 6,38 & 6,41 & 5,74 & 5,13 & 5,82 & 6,13 & 121,7 & 145,8 & 114,5 & 108,0 \\
\hline 3031 & 6,56 & 7,29 & 6,18 & 6,22 & 6,13 & 6,02 & 6,22 & 6,13 & 114,8 & 129,3 & 110,1 & 105,0 \\
\hline Promedio & 7,04 & 8,12 & 6,91 & 6,10 & 6,02 & 5,92 & 6,09 & 6,04 & 122,2 & 142,8 & 119,6 & 104,5 \\
\hline DMS & 0,40 & 0,74 & 0,69 & 0,66 & 0,14 & 0,32 & 0,17 & 0,20 & 6,31 & 10,9 & 11,6 & 10,6 \\
\hline
\end{tabular}

loc. $=$ localidades, $\mathrm{Gr}=$ grupo.

observan en el Cuadro 8. Todos los híbridos son muy similares en su precocidad con excepción del híbrido 30P-70, cuya floración promedio fue de 52 dds. Con respecto a la altura de las plantas, el híbrido de menor tamaño fue el 30K-75 con $219 \mathrm{~cm}$ de altura, seguido por los híbridos 30F-80 y 30S-40. Las medidas de la altura de la mazorca variaron entre 113 a $147 \mathrm{~cm}$, siendo los híbridos 30P-70 y 30F-87 los híbridos con la posición más baja de esta característica.

En la evaluación del porcentaje de plantas acamadas se detectaron diferencias altamente significativas entre los híbridos evaluados. Los híbridos 30F-80, P-0408, P-0512, 30K-75 y DK-466 sobresalieron por presentar los valores más bajos (menores a 15\%). Los híbridos PB-0103 y PB-0105 presentaron alta susceptibilidad al acame de tallo con porcentajes superiores al $35 \%$.

En cuanto a las principales enfermedades foliares (Curvularia sp y Puccinia sp.), no se observaron diferencias significativas entre los híbridos evaluados. De acuerdo a la evaluación en campo, ninguno de los híbridos presentó una calificación superior a 3,0, lo que sugiere que en este año, las enfermedades no se presentaron en intensidades suficientes para ser una limitante en el desarrollo del cultivo. La otra enfermedad causante de pérdidas en el cultivo, lo es el complejo de hongos que ataca la mazorca (Diplodia maydis y Fusarium sp); los cuales se presentaron en bajos porcentajes. Los híbridos 30S-40 y 30F-80 presentaron los valores más bajos ( 0,9 y $1,0 \%$, respectivamente), mientras que los porcentajes más altos se obtuvieron con los híbridos 30R-92 con 4,7\% y 3031 con 3,8 (Cuadro 8).

\section{Interacción Genotipo por Ambiente}

El Cuadro 9 muestra el análisis de varianza del rendimiento de grano, así como el valor de los dos ejes principales de la interacción genotipo-ambiente, obtenidos a través del modelo AMMI Biplot GGE-SREG. El resultado indicó que los dos primeros ejes (PCA) explicaron el 63,6\% de la interacción genotipo ambiente con tan solo el 36\% de los grados de libertad. El PCA-1 explicó el 39,9\%, mientras que el PCA-2 fue responsable del $23,7 \%$ con el 19 y $17 \%$ de los grados de libertad, respectivamente.

En el Cuadro 10 se presentan las puntuaciones o valores AMMI, de los 14 genotipos y de los nueve ambientes, los cuales presentan diferentes patrones de interacción. De acuerdo a las puntuaciones de ambos ejes (PCA-1 y PCA-2) el híbrido más estable fue P-0102, siendo el híbrido nacional PB-0105 el que mejor respondió a las condiciones ambientales prevalecientes durante el desarrollo del cultivo, presentando el mejor 
Cuadro 8. Características agronómicas de la Prueba Regional de maíz según híbridos, Panamá, 2005.

\begin{tabular}{|c|c|c|c|c|c|c|c|c|c|c|}
\hline Híbridos & $\begin{array}{l}\text { Flor } \\
\text { (días) }\end{array}$ & $\begin{array}{c}\text { Altura } \\
\text { planta } \\
(\mathbf{c m})\end{array}$ & $\begin{array}{c}\text { Altura } \\
\text { mazorca } \\
\text { (cm) }\end{array}$ & $\begin{array}{l}\text { Mazorcas/ } \\
\mathbf{m}^{2}\end{array}$ & $\begin{array}{c}\text { Mazor- } \\
\text { cas/ } \\
\text { planta }\end{array}$ & $\begin{array}{c}\text { Mazorcas } \\
\text { podridas } \\
(\%)\end{array}$ & $\begin{array}{c}\text { Acame } \\
(\%)\end{array}$ & $\begin{array}{c}\text { Roya } \\
(1-5)\end{array}$ & $\begin{array}{c}\text { Curvularia } \\
\qquad(1-5)\end{array}$ & $\begin{array}{c}\text { Altura } \\
\text { mazorca } \\
\text { Altura } \\
\text { planta }\end{array}$ \\
\hline $30 F-80$ & 56 & 234 & 127 & 5,85 & 0,96 & 1,0 & 3,0 & 2,3 & 2,1 & 0,54 \\
\hline $30 \mathrm{~K}-75$ & 54 & 219 & 118 & 5,89 & 0,96 & 2,1 & 13,8 & 2,4 & 2,1 & 0,54 \\
\hline $30 \mathrm{P}-70$ & 52 & 231 & 113 & 6,02 & 0,98 & 2,1 & 20,8 & 2,8 & 2,7 & 0,49 \\
\hline $30 \mathrm{~S}-40$ & 56 & 234 & 131 & 5,93 & 0,97 & 0,9 & 21,5 & 2,9 & 2,3 & 0,56 \\
\hline 3031 & 55 & 251 & 128 & 5,73 & 0,93 & 3,8 & 14,7 & 2,4 & 2,2 & 0,51 \\
\hline $30 F-87$ & 54 & 224 & 121 & 5,87 & 0,97 & 1,5 & 17,0 & 2,8 & 2,4 & 0,54 \\
\hline 30R-92 & 56 & 240 & 131 & 5,53 & 0,96 & 4,7 & 19,0 & 2,9 & 2,4 & 0,55 \\
\hline PB-0103 & 57 & 251 & 134 & 5,71 & 0,96 & 2,6 & 39,3 & 2,3 & 2,1 & 0,53 \\
\hline PB-0105 & 55 & 248 & 137 & 5,88 & 0,96 & 2,2 & 36,1 & 2,4 & 2,1 & 0,55 \\
\hline P-0102 & 58 & 254 & 147 & 5,72 & 0,97 & 1,9 & 17,7 & 2,4 & 2,1 & 0,58 \\
\hline P-0408 & 56 & 253 & 139 & 5,55 & 0,92 & 1,6 & 8,8 & 2,6 & 2,2 & 0,55 \\
\hline P-0512 & 56 & 239 & 128 & 5,80 & 0,95 & 2,4 & 11,1 & 2,9 & 2,3 & 0,53 \\
\hline DK-466 & 55 & 243 & 132 & 5,72 & 0,96 & 2,5 & 12,1 & 2,9 & 2,4 & 0,54 \\
\hline P-0104 & 55 & 245 & 134 & 5,70 & 0,98 & 3,2 & 28,9 & 2,6 & 2,2 & 0,55 \\
\hline Promedio & 55 & 240 & 129 & 5,77 & 0,96 & 2,3 & 18,8 & 2,6 & 2,3 & 0,54 \\
\hline DMS & 0,6 & 5,4 & 4,5 & 0,18 & 0,02 & 1,2 & 5,7 & 0,15 & 0,13 & 0,02 \\
\hline
\end{tabular}

rendimiento. El híbrido 30S-40, presentó el mejor comportamiento en las localidades del grupo B.

De acuerdo a Yan et al. (2000), al graficar las puntuaciones de ambos ejes principales (PCA1 y PCA2), se forma un polígono con los híbridos que quedan en la parte externa de la figura (éstos fueron los híbridos P0512, 30S-40, P-0512, 30R-92, y 30F-87). Los híbridos localizados en los vértices son considerados los mejores e inferiores dependiendo de su ubicación. Con relación a la interacción genotipo ambiente la Figura 1, muestra los híbridos que mejor se comportaron en cada uno de los grupos ambientales, de acuerdo a la posición o cercanía a la que se encuentran de cada grupo. Ejemplo de esto lo observamos con el híbrido PB-0105 que tuvo buen comportamiento en las localidades del Grupo $\mathrm{A}$ y $\mathrm{C}$ pero fue un tanto menor en las localidades del Grupo B, mientras que el 30S-40 se comportó mejor en los ambientes del Grupo A y B pero bajó su rendimiento en el Grupo C. La Figura 1, muestra que Las Cabras (CAB) en el Grupo C y Mariabé (MAR) en el Grupo A,
Cuadro 9. Análisis de varianza Tipo IV y componentes principales (PCA) para la variable rendimiento de grano de la Prueba Regional de maíz, Azuero, Panamá, 2005.

\begin{tabular}{|c|c|c|c|c|}
\hline $\begin{array}{l}\text { Fuente de } \\
\text { variación }\end{array}$ & G.L. & $\begin{array}{c}\text { Suma de } \\
\text { cuadrados } \\
\text { Tipo IV }\end{array}$ & $\begin{array}{c}\text { Cuadrados } \\
\text { medios }\end{array}$ & Prob > F \\
\hline Ambiente (A) & 8 & 331,732 & 41,466 & 0,001 \\
\hline Genotipo (G) & 13 & 51,487 & 3,961 & 0,001 \\
\hline $\mathrm{G} \times \mathrm{A}$ & 104 & 140,103 & 1,347 & 0,001 \\
\hline PCA-1 & 20 & 57,507 & 2,875 & 0,001 \\
\hline PCA-2 & 18 & 25,693 & 1,427 & 0,001 \\
\hline Residuo & 66 & 62,903 & 0,953 & 0,001 \\
\hline
\end{tabular}

fueron los ambientes que mejor discriminaron los genotipos. Mientras, las localidades del Grupo B, tuvieron la misma capacidad para discriminar a los genotipos evaluados. 
Cuadro 10. Puntuaciones de los dos ejes correspondientes a los componentes principales (PCA) para la variable rendimiento de grano según genotipo y localidad, Azuero, Panamá, 2005.

\begin{tabular}{|c|c|c|c|c|c|c|c|c|c|}
\hline \multicolumn{2}{|l|}{ Híbrido } & \multirow{2}{*}{$\begin{array}{r}\begin{array}{r}\text { Rend } \\
(\mathbf{t} / \mathbf{h a})\end{array} \\
7,16\end{array}$} & \multirow{2}{*}{$\begin{array}{c}\begin{array}{c}\text { Puntuación } \\
\text { PCA-1 }\end{array} \\
-0,054\end{array}$} & \multirow{2}{*}{$\begin{array}{c}\begin{array}{c}\text { Puntuación } \\
\text { PCA-2 }\end{array} \\
0,727\end{array}$} & \multicolumn{2}{|l|}{ Localidad } & \multirow{2}{*}{$\begin{array}{r}\begin{array}{r}\text { Rend } \\
\text { (t/ha) }\end{array} \\
6,87\end{array}$} & $\begin{array}{c}\text { Puntuación } \\
\text { PCA-1 }\end{array}$ & \multirow{2}{*}{$\begin{array}{c}\text { Puntuación } \\
\text { PCA-2 } \\
-0,367\end{array}$} \\
\hline $30 F-80$ & (F80) & & & & El Ejido & (EJ) & & $-0,584$ & \\
\hline $30 \mathrm{~K}-75$ & (K75) & 6,91 & 0,039 & 0,329 & La Enea & (ENE) & 7,18 & $-0,328$ & 0,988 \\
\hline $30 \mathrm{P}-70$ & (P70) & 6,77 & 0,057 & $-0,315$ & Las Cocobolas & $(\mathrm{Coc})$ & 6,68 & $-0,484$ & 0,475 \\
\hline $30 \mathrm{~S}-40$ & (S40) & 7,38 & $-1,106$ & $-0,655$ & Paraíso & (PAR) & 7,36 & 0,050 & $-0,558$ \\
\hline 3031 & (X31) & 6,56 & 0,645 & $-0,028$ & Guararé & (GUA) & 6,53 & 0,676 & 0,795 \\
\hline $30 F-87$ & (F87) & 6,73 & $-0,517$ & 0,211 & Pocrí & (PoC) & 8,06 & $-0,664$ & $-0,222$ \\
\hline 30R-92 & (R92) & 6,59 & 0,896 & 0,243 & Las Cabras & $(\mathrm{CAB})$ & 6,07 & 1,354 & $-0,074$ \\
\hline PB-0103 & (P03) & 7,30 & 0,140 & $-0,803$ & Mariabé & (MAR) & 8,94 & $-0,703$ & $-0,354$ \\
\hline PB-0105 & (P05) & 8,01 & 0,044 & $-0,259$ & Sabana Grande & (SGD) & 5,72 & 0,683 & $-0,683$ \\
\hline P-0102 & (P02) & 7,19 & $-0,434$ & 0,686 & & & & & \\
\hline P-0408 & (P08) & 6,79 & 0,688 & 0,087 & & & & & \\
\hline P-0512 & (P12) & 7,24 & 0,651 & $-0,467$ & & & & & \\
\hline DK-466 & (DK6) & 7,10 & $-0,560$ & $-0,208$ & & & & & \\
\hline P-0104 & (P04) & 6,88 & $-0,489$ & 0,454 & & & & & \\
\hline
\end{tabular}

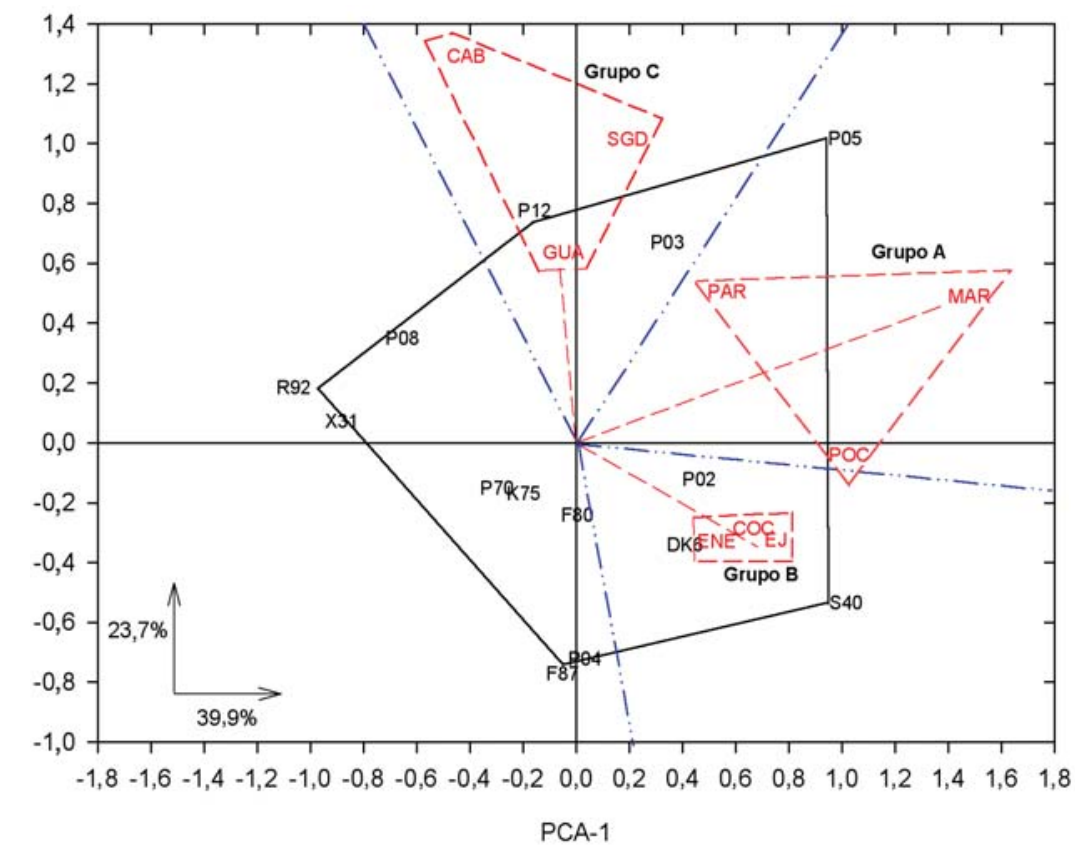

Figura 1. Puntuaciones del primer y segundo eje del componente principal de 14 cultivares de maíz en nueve ambientes de Panamá (Biplot GGE-SREG). 


\section{CONCLUSIONES}

PB-0105 de grano blanco fue el híbrido más rendidor; 30S-40 de grano amarillo, PB-0103 de grano blanco, P-0512, P-0102, 30F-80 y DK-466 de grano amarillo, también presentaron alto rendimiento (mayor a 7,0 t/ha) y buenas características agronómicas.

El modelo Biplot GGE-SREG, identificó a los híbridos PB-0105 y 30S-40 como los que mejor respondieron a los estímulos ambientales y al P0102 como el de mejor estabilidad.

Bajo las condiciones climáticas del año de estudio, la presencia o no de estrés hídrico en la época entre los 50 a 80 dds y más de 80 dds en algunas localidades, el modelo Biplot GGE-SREG logró identificar tres grupos ambientales.

PB-0105, PB-0103 y P-0512 sobresalieron en las localidades con estrés hídrico, mientras que PB-0103 y 30S-40 fueron los mejores híbridos en las localidades sin estrés.

\section{LITERATURA CITADA}

BECKER, H.C.; LEON, J. 1988. Stability analysis in plant breeding. Plant Breeding 101:1-23.

BECKER, H.C. 1981. Correlation among some statistical measure of phenotypic stability. Euphytica 30: 835-840.

CROSSA, J. 1988. A comparison of results obtained with two methods for assessing yield stability. Theor. Appl. Genet 75: 460- 467.

CROSSA, J. 1990. Statistical analysis of multi location trials. Advances in Agronomy 44: 55-85.

CROSSA, J.; GAUCH, JR., H.G.; ZOBEL, R.W. 1990. Additive main effects and multiplicative interaction analysis of two international maize cultivar trials. Crop. Sci. 30:493-500.

CROSSA, J.; FOX, P.N.; PFEIFFER, W.H.; RAJARAM, S.; GAUCH, JR., H.G. 1991. AMMI adjustment for statistical analysis of an international wheat yield trial. Theor Appl. Genet. 81:27-37.

DÍAZ-ROMEU, R.; HUNTER, A. 1978. Metodología de muestreo de suelos y tejidos vegetal e investigación en invernadero. Turrialba, Costa Rica, CATIE. 68 p.

EBERHART, S. A.; RUSSELL, W.A. 1966. Stability parameters for comparing varieties. Crop. Sci. 6:36-40.

FINLAY, K.W.; WILKINSON, G.N. 1963. The analysis of adaptation in plant breeding program. Aust. J. Agric. Res. 14: 742-754.

GAUCH, H. G.; ZOBEL, R.W. 1988. Predictive and postdictive success of statistical analysis of yield trials. Theor. Appl. Genet. 76:1-10.

GORDÓN. R.; CAMARGO, I.; FRANCO, J.; GONZÁLEZ, A. 2004. Impacto de la precipitación pluvial en el rendimiento de grano de maíz en la región de Azuero, Panamá, 1995-2003. I. Análisis de la distribución de lluvias y su relación con la época de siembra. Revista Ciencia Agropecuaria No. 16 IDIAP. 17-30.

LIN, C.S.; BINNS, M.R.; LEFKOVITH, L.P. 1986. Stability Analysis. Where do we stand? Crop Sci. 26.894-900.

MARQUEZ, F. 1991. Genotecnia vegetal "métodos, teorías resultados". Primera Edición. México. 500 p.

PERKINS, J. M.; JINKS, J.L. 1968. Environmental and genotype-environmental components of variability. IV Non-linear interactions for multiple inbred lines. Heredity 23:525-535.

WESTCOTT, B. 1986. Some methods of analyzing genotype environment interaction. Heredity 56: 243-253.

YAN, W. L.; HUNT, A.; SHENG, Q.; SZLAVNICS, Z. 2000. Cultivar evaluation and mega environmentinvestigation based on the GGE Biplot. Crop Sci. 40:597-605.

ZOBEL R.W.; WRIGHT, M.J.; GAUCH JR., H.G. 1988. Statistical analysis of a yield trial. Agron. J. 80:388-393. 